\title{
SLIDING MODE CONTROL OF A MAGNETIC LEVITATION SYSTEM
}

\author{
N. F. AL-MUTHAIRI AND M. ZRIBI
}

Received 18 October 2003 and in revised form 23 February 2004

Sliding mode control schemes of the static and dynamic types are proposed for the control of a magnetic levitation system. The proposed controllers guarantee the asymptotic regulation of the states of the system to their desired values. Simulation results of the proposed controllers are given to illustrate the effectiveness of them. Robustness of the control schemes to changes in the parameters of the system is also investigated.

\section{Introduction}

Magnetic levitation systems have practical importance in many engineering systems such as in high-speed maglev passenger trains, frictionless bearings, levitation of wind tunnel models, vibration isolation of sensitive machinery, levitation of molten metal in induction furnaces, and levitation of metal slabs during manufacturing. The maglev systems can be classified as attractive systems or repulsive systems based on the source of levitation forces. These kind of systems are usually open-loop unstable and are described by highly nonlinear differential equations which present additional difficulties in controlling these systems. Therefore, it is an important task to construct high-performance feedback controllers for regulating the position of the levitated object.

In recent years, a lot of works have been reported in the literature for controlling magnetic levitation systems. The feedback linearization technique has been used to design control laws for magnetic levitation systems $[2,9,30]$. The input-output, input-state, and exact linearization techniques have been used to develop nonlinear controllers $[6,11,38]$. Other types of nonlinear controllers based on nonlinear methods have been reported in the literature $[14,18,35,40]$. Robust linear controller methods such as $H_{\infty}$ optimal control, $\mu$-synthesis, and $Q$-parameterization have also been applied to control magnetic levitation systems [12, 13, 23]. Control laws based on phase space [39], linear controller design [10], the gain scheduling approach [21], and neural network techniques [22] have also been used to control magnetic levitation systems.

During the last two decades, variable structure systems (VSS) and sliding mode control (SMC) have received significant interest and have become well-established research areas 
with great potential for practical applications. The theoretical development aspects of SMC are well documented in many books and articles [3, 19, 29, 31, 32, 33, 34, 37, 41, 42]. The discontinuous nature of the control action in SMC is claimed to result in outstanding robustness features for both system stabilization and output tracking problems. The very good performance also includes insensitivity to parameter variations and rejection of disturbances. VSS has been applied in many control fields which include robot control [36], motor control [15], flight control [17], control of power systems [4], and process control [20]. In addition, SMC has been used in magnetic bearing systems $[1,24,25]$; however, the proposed controllers have been designed based on linearized models about nominal operating points, and thus the tracking performance deteriorates rapidly with increasing deviations from the nominal operating points.

One of the first applications of SMC to magnetic levitation systems was carried out by Cho et al. [8]. They showed that a sliding mode controller provides better transient response than classical controllers. However, they neglected the current dynamics in their model and limited the ball's motion to a range of $1 \mathrm{~mm}$. Chen et al. [7] designed an adaptive sliding mode controller for a rather different type of magnetic levitation systems called dual-axis maglev positioning system. Buckner [5] introduced a procedure for estimating the uncertainty bounds using artificial neural network and then applied it to SMC of a magnetic levitation system. Hassan and Mohamed [16] used the reaching law method complemented with the sliding mode equivalence technique to design a variable structure controller for the magnetic levitation system.

In this paper, we propose one static and two dynamic SMC schemes for the magnetic levitation system. The proposed controllers are based on the SMC schemes developed by Sira-Ramírez et al. [27, 28] and Sira-Ramírez [26]; these control schemes have been shown to enjoy advantageous insensitivity with respect to variations in the system's parameters and to external perturbations. Simulation results indicate that the proposed control schemes work well and are robust to changes in the system's parameters.

The rest of the paper is organized as follows. Section 2 contains the mathematical model of the magnetic levitation system. Section 3 deals with the design of a static SMC for the magnetic levitation system. Sections 4 and 5 deal with the design of dynamic sliding mode controllers for the system. Section 6 presents and discusses the simulation results of the proposed control schemes. Finally, the conclusion is given in Section 7.

\section{Model of the magnetic levitation system}

The magnetic levitation system considered in this paper consists of a ferromagnetic ball suspended in a voltage-controlled magnetic field. Only the vertical motion is considered. The objective is to keep the ball at a prescribed reference level. The schematic diagram of the system is shown in Figure 2.1. The dynamic model of the system can be written as [2]

$$
\begin{gathered}
\frac{d p}{d t}=v, \quad R i+\frac{d(L(p) i)}{d t}=e, \\
m \frac{d v}{d t}=m g_{c}-C\left(\frac{i}{p}\right)^{2},
\end{gathered}
$$




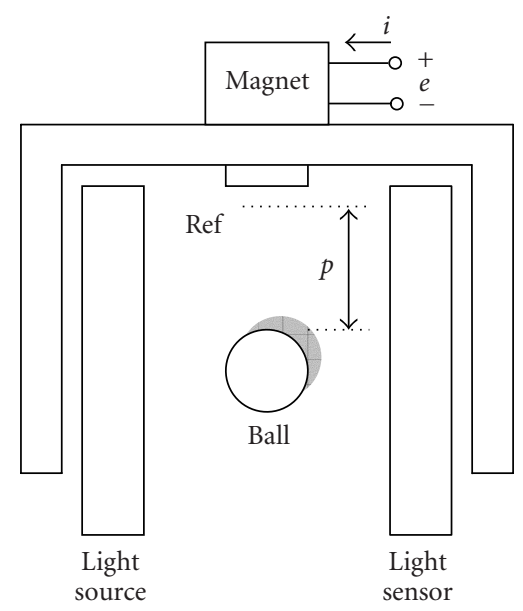

Figure 2.1. Schematic diagram of the magnetic levitation system.

where $p$ denotes the ball's position, $v$ is the ball's velocity, $i$ is the current in the coil of the electromagnet, $e$ is the applied voltage, $R$ is the coil's resistance, $L$ is the coil's inductance, $g_{c}$ is the gravitational constant, $C$ is the magnetic force constant, and $m$ is the mass of the levitated ball.

The inductance $L$ is a nonlinear function of the ball's position $p$. The approximation

$$
L(p)=L_{1}+\frac{2 C}{p}
$$

will be used; $L_{1}$ is a parameter of the system.

Let the states and the control input be chosen such that $x_{1}=p, x_{2}=v, x_{3}=i, u=e$, and $\mathbf{x}=\left(\begin{array}{lll}x_{1} & x_{2} & x_{3}\end{array}\right)^{T}$ is the state vector. Thus, the state-space model of the magnetic levitation system can be written as

$$
\begin{gathered}
\frac{d x_{1}}{d t}=x_{2}, \\
\frac{d x_{2}}{d t}=g_{c}-\frac{C}{m}\left(\frac{x_{3}}{x_{1}}\right)^{2}, \\
\frac{d x_{3}}{d t}=-\frac{R}{L} x_{3}+\frac{2 C}{L}\left(\frac{x_{2} x_{3}}{x_{1}^{2}}\right)+\frac{1}{L} u .
\end{gathered}
$$

The state-space model of the magnetic levitation system (2.3) will be used in the design of the SMC schemes.

Let $x_{1 d}, x_{2 d}$, and $x_{3 d}$ be the desired values of $x_{1}, x_{2}$, and $x_{3}$, respectively. Note, from (2.3), that the equilibrium point for the system is $\mathbf{x}_{e}=\left(\begin{array}{lll}x_{1 e} & 0 & x_{3 e}\end{array}\right)^{T}$, where $x_{3 e}$ satisfies $x_{3 e}=\sqrt{g_{c} m / C} x_{1 e}$. Therefore, one may conclude that $x_{2 d}$ is equal to zero.

The objective of the control schemes is to drive the states $x_{1}, x_{2}$, and $x_{3}$ to their desired constant values $x_{1 d}, x_{2 d}$, and $x_{3 d}$, respectively. 
Now, consider the following nonlinear change of coordinates:

$$
\begin{gathered}
z_{1}=x_{1}-x_{1 d}, \\
z_{2}=x_{2}, \\
z_{3}=g_{c}-\frac{C}{m}\left(\frac{x_{3}}{x_{1}}\right)^{2} .
\end{gathered}
$$

Remark 2.1. If $z_{1}, z_{2}, z_{3}$ are driven to zero as $t \rightarrow \infty$, then $x_{1}$ will converge to $x_{1 d}, x_{2}$ will converge to zero, and $x_{3}$ will converge to $x_{3 d}=\sqrt{g_{c} m / C} x_{1 d}$ as $t \rightarrow \infty$.

The dynamic model of the magnetic levitation system in the new coordinates system can be written as

$$
\begin{gathered}
\dot{z}_{1}=z_{2}, \\
\dot{z}_{2}=z_{3}, \\
\dot{z}_{3}=f(\mathbf{z})+g(\mathbf{z}) u,
\end{gathered}
$$

where

$$
\begin{gathered}
f(\mathbf{z})=2\left(g_{c}-z_{3}\right)\left(\left(1-\frac{2 C}{L\left(z_{1}+x_{1 d}\right)}\right) \frac{z_{2}}{\left(z_{1}+x_{1 d}\right)}+\frac{R}{L}\right), \\
g(\mathbf{z})=\frac{-2}{L\left(z_{1}+x_{1 d}\right)} \sqrt{\frac{C}{m}\left(g_{c}-z_{3}\right) .}
\end{gathered}
$$

It should be noted that the functions $f(\mathbf{z})$ and $g(\mathbf{z})$ correspond in the original coordinates to the following functions, respectively:

$$
\begin{gathered}
f_{1}(\mathbf{x})=\frac{2 C}{m}\left(\left(1-\frac{2 C}{L x_{1}}\right) \frac{x_{2} x_{3}^{2}}{x_{1}^{3}}+\frac{R}{L} \frac{x_{3}^{2}}{x_{1}^{2}}\right), \\
g_{1}(\mathbf{x})=-\frac{2 C x_{3}}{L m x_{1}^{2}}
\end{gathered}
$$

where $f_{1}(\mathbf{x})=f(\mathbf{z})$ and $g_{1}(\mathbf{x})=g(\mathbf{z})$.

Let the output of the system be

$$
y=z_{1}=x_{1}-x_{1 d}
$$

Using (2.5), (2.7), and (2.8), the relationship between the input and the output of the system can be found as

$$
y^{(3)}=f_{1}(\mathbf{x})+g_{1}(\mathbf{x}) u \text {. }
$$

Using model (2.5), (2.6), (2.7), (2.8), and (2.9), the design of SMC schemes for the magnetic levitation system will be considered in the next sections. 


\section{Design of a static sliding mode control}

The design of a static SMC scheme for the magnetic levitation system is discussed in this section.

The first step in designing an SMC scheme for the system is to design the switching surface. Let the switching surface $\mathscr{S}$ be

$$
\mathscr{S}=\ddot{y}+\lambda_{1} \dot{y}+\lambda_{2} y=\ddot{z}_{1}+\lambda_{1} \dot{z}_{1}+\lambda_{2} z_{1}=z_{3}+\lambda_{1} z_{2}+\lambda_{2} z_{1}
$$

where $\lambda_{1}$ and $\lambda_{2}$ are positive scalars.

Using (2.4), the switching surface $\mathscr{Y}$ can be written as a function of $x_{1}, x_{2}$, and $x_{3}$ such that

$$
\mathscr{S}=g_{c}-\frac{C}{m}\left(\frac{x_{3}}{x_{1}}\right)^{2}+\lambda_{1} x_{2}+\lambda_{2}\left(x_{1}-x_{1 d}\right) .
$$

Note that the choice of the switching surface guarantees that $y=z_{1}=x_{1}-x_{1 d}$ converges to 0 as $t \rightarrow \infty$ when we have sliding (i.e., $\mathscr{S}=0$ ).

The following proposition gives the first result of the paper.

Proposition 3.1. The discontinuous static feedback controller,

$$
\begin{aligned}
u=\frac{1}{g_{1}}[ & -f_{1}-\lambda_{1}\left(g_{c}-\frac{C}{m}\left(\frac{x_{3}}{x_{1}}\right)^{2}\right)-\lambda_{2} x_{2} \\
& \left.-W \operatorname{sign}\left(g_{c}-\frac{C}{m}\left(\frac{x_{3}}{x_{1}}\right)^{2}+\lambda_{1} x_{2}+\lambda_{2}\left(x_{1}-x_{1 d}\right)\right)\right],
\end{aligned}
$$

when applied to the magnetic levitation system (2.3), asymptotically stabilizes $x_{1}, x_{2}$, and $x_{3}$ to their desired values as $t \rightarrow \infty$.

Proof. Differentiating (3.1) with respect to time and using (2.5), (2.6), (2.7), (2.8), and (2.9), we can write the following:

$$
\dot{\mathscr{S}}=y^{(3)}+\lambda_{1} \ddot{y}+\lambda_{2} \dot{y}=f_{1}(\mathbf{x})+g_{1}(\mathbf{x}) u+\lambda_{1} z_{3}+\lambda_{2} z_{2} .
$$

Substituting $u$ by its value from (3.3), it follows that

$$
\begin{aligned}
\dot{\mathscr{S}}=f_{1}+\lambda_{1} z_{3}+\lambda_{2} z_{2}+ & {\left[-f_{1}-\lambda_{1}\left(g_{c}-\frac{C}{m}\left(\frac{x_{3}}{x_{1}}\right)^{2}\right)-\lambda_{2} x_{2}\right.} \\
& \left.\quad-W \operatorname{sign}\left(g_{c}-\frac{C}{m}\left(\frac{x_{3}}{x_{1}}\right)^{2}+\lambda_{1} x_{2}+\lambda_{2}\left(x_{1}-x_{1 d}\right)\right)\right] \\
= & -W \operatorname{sign}\left(g_{c}-\frac{C}{m}\left(\frac{x_{3}}{x_{1}}\right)^{2}+\lambda_{1} x_{2}+\lambda_{2}\left(x_{1}-x_{1 d}\right)\right) \\
= & -W \operatorname{sign}(\mathscr{S}) .
\end{aligned}
$$


The dynamics in (3.5) guarantees the finite-time reachability of $\mathscr{S}$ to zero from any given initial condition $\mathscr{S}(0)$ provided that the constant gain $W$ is chosen to be strictly positive. Moreover, the dynamics in (3.5) guarantees that $\mathscr{S} \dot{\mathscr{S}}<0$ (the condition needed to guarantee switching).

Since $\mathscr{T}$ is driven to zero in finite time, the output $y=z_{1}$ is governed after such finite amount of time by the second-order differential equation $\ddot{y}+\lambda_{1} \dot{y}+\lambda_{2} y=0$. Thus the output $y(t)=z_{1}(t)$ will converge asymptotically to 0 as $t \rightarrow \infty$ because $\lambda_{1}$ and $\lambda_{2}$ are positive scalars. Since $z_{1}$ converges to zero, then $z_{2}$ and $z_{3}$ will converge to zero as $t \rightarrow \infty$. Thus $x_{1}, x_{2}$, and $x_{3}$ will also converge to their desired values as $t \rightarrow \infty$.

Therefore, it can be concluded that the static sliding mode controller given by (3.3) guarantees the asymptotic convergence of the states $x_{1}, x_{2}$, and $x_{3}$ to their desired values as $t \rightarrow \infty$.

Remark 3.2. Like any other variable structure controller, the proposed controller is confronted with the problem of chattering, which is undesirable in practice. To cope with this problem, the boundary layer concept (see [29]) or dynamic SMC schemes can be used.

\section{Design of a dynamic sliding mode control}

To reduce the chattering due to the static sliding mode controller, a dynamic sliding mode controller is proposed in this section.

Differentiating (2.9) with respect to time, it follows that

$$
y^{(4)}=\dot{f}_{1}+\dot{g_{1}} u+g_{1} \dot{u},
$$

where

$$
\begin{gathered}
\dot{f}_{1}(\mathbf{x})=\frac{2 C}{m}\left[\frac{-2 R^{2}}{L^{2}} \frac{x_{3}^{2}}{x_{1}^{2}}+\left(g_{c}-\frac{4 R}{L} x_{2}\right) \frac{x_{3}^{2}}{x_{1}^{3}}+\left(\frac{10 R C}{L^{2}} x_{2}-3 x_{2}^{2}-\frac{2 C g_{c}}{L}\right) \frac{x_{3}^{2}}{x_{1}^{4}}\right. \\
+\left(\frac{12 C}{L} x_{2}^{2}-\frac{C}{m} x_{3}^{2}\right) \frac{x_{3}^{2}}{x_{1}^{5}}+\left(\frac{2 C^{2}}{L m} x_{3}^{2}-\frac{12 C^{2}}{L^{2}} x_{2}^{2}\right) \frac{x_{3}^{2}}{x_{1}^{6}} \\
\left.+\left(\frac{2}{L} \frac{x_{2}}{x_{1}}-\frac{4 C}{L^{2}} \frac{x_{2}}{x_{1}^{2}}+\frac{2 R}{L^{2}}\right) \frac{x_{3}}{x_{1}^{2}} u\right], \\
\dot{g}_{1}(\mathbf{x})=\left(\frac{-R}{L}-\frac{2 x_{2}}{x_{1}}+\frac{4 C}{L} \frac{x_{2}}{x_{1}^{2}}\right) g_{1}(x)-\frac{2 C}{m L^{2} x_{1}^{2}} u .
\end{gathered}
$$

To design the dynamic sliding mode controller, we will choose the switching surface $\sigma$ such that

$$
\sigma=y^{(3)}+m_{1} \ddot{y}+m_{2} \dot{y}+m_{3} y
$$

where $m_{1}, m_{2}$, and $m_{3}$ are parameters to be chosen by the designer such that the polynomial $p_{1}(s)=s^{3}+m_{1} s^{2}+m_{2} s+m_{3}$ is a Hurwitz polynomial. 
Using (2.4) and (2.9), the switching surface $\sigma$ can be written as

$$
\sigma=f_{1}+g_{1} u+m_{1}\left(g_{c}-\frac{C}{m}\left(\frac{x_{3}}{x_{1}}\right)^{2}\right)+m_{2} x_{2}+m_{3}\left(x_{1}-x_{1 d}\right)
$$

The following proposition gives the second result of the paper.

Proposition 4.1. The dynamic control scheme,

$$
\dot{u}=\frac{1}{g_{1}}\left[-\dot{f}_{1}-\dot{g_{1}} u-m_{1}\left(f_{1}+g_{1} u\right)-m_{2}\left(g_{c}-\frac{C}{m}\left(\frac{x_{3}}{x_{1}}\right)^{2}\right)-m_{3} x_{2}-\Gamma \operatorname{sign}(\sigma)\right],
$$

when applied to the magnetic levitation system (2.3), asymptotically stabilizes the states to their desired values as $t \rightarrow \infty$.

Proof. Differentiating (4.3) with respect to time and using (2.4), (2.5), (2.8), and (4.1), it follows that

$$
\begin{aligned}
\dot{\sigma} & =y^{(4)}+m_{1} y^{(3)}+m_{2} \ddot{y}+m_{3} \dot{y} \\
& =\dot{f}_{1}+\dot{g_{1}} u+g_{1} \dot{u}+m_{1}\left(f_{1}+g_{1} u\right)+m_{2}\left(g_{c}-\frac{C}{m}\left(\frac{x_{3}}{x_{1}}\right)^{2}\right)+m_{3} x_{2} .
\end{aligned}
$$

Substituting $\dot{u}$ by its value from (4.5), we get

$$
\begin{aligned}
\dot{\sigma}= & \dot{f}_{1}+\dot{g}_{1} u+m_{1}\left(f_{1}+g_{1} u\right)+m_{2}\left(g_{c}-\frac{C}{m}\left(\frac{x_{3}}{x_{1}}\right)^{2}\right)+m_{3} x_{2} \\
& +\left[-\dot{f}_{1}-\dot{g_{1}} u-m_{1}\left(f_{1}+g_{1} u\right)-m_{2}\left(g_{c}-\frac{C}{m}\left(\frac{x_{3}}{x_{1}}\right)^{2}\right)-m_{3} x_{2}-\Gamma \operatorname{sign}(\sigma)\right] \\
= & -\Gamma \operatorname{sign}(\sigma) .
\end{aligned}
$$

The dynamics in (4.7) guarantees the finite-time reachability of $\sigma$ to zero from any given initial condition $\sigma(0)$ provided that the constant gain $\Gamma$ is chosen to be strictly positive. Moreover, the dynamics in (4.7) guarantees that $\sigma \dot{\sigma}<0$ (the condition needed to guarantee switching).

Since $\sigma$ is driven to zero in finite time, the output $y=z_{1}$ is governed after such finite amount of time by the third-order differential equation $y^{(3)}+m_{1} \ddot{y}+m_{2} \dot{y}+m_{3} y=0$. Thus the output $y(t)=z_{1}$ will converge to zero as $t \rightarrow \infty$ because $m_{1}, m_{2}$, and $m_{3}$ are positive scalars chosen such that the polynomial $p_{1}(s)=s^{3}+m_{1} s^{2}+m_{2} s+m_{3}$ is a Hurwitz polynomial. Since $z_{1}$ converges to zero, then $z_{2}$ and $z_{3}$ will converge to zero as $t \rightarrow \infty$. Thus $x_{1}, x_{2}$, and $x_{3}$ will also converge to their desired values as $t \rightarrow \infty$.

Therefore, it can be concluded that the dynamic sliding mode controller given by (4.5) guarantees the asymptotic convergence of the states $x_{1}, x_{2}$, and $x_{3}$ to their desired values. 
The controller developed in this section needs the computation of the derivatives of the system's dynamics. However, the computation of the derivatives might be problematic. Hence, a dynamic SMC scheme that does not require the computation of the derivatives of the system's dynamics is proposed in the next section.

\section{Design of a modified dynamic sliding mode control}

Sira-Ramírez et al. [28] proposed the use of a robust redundant feedback controller, based on dynamical sliding mode control, for nonlinear systems for which a smooth feedback control policy is available. Motivated by this work, a modified dynamic sliding mode controller is now designed for the magnetic levitation system.

Recall that the dynamic model of the magnetic levitation system in the $z$-coordinates system can be written as (2.5), where the output of the system is chosen as

$$
y=z_{1}
$$

It can be shown that the feedback linearization controller

$$
u=-\frac{1}{g}\left(f+c_{1} z_{1}+c_{2} z_{2}+c_{3} z_{3}\right)
$$

guarantees the asymptotic convergence of $z_{1}, z_{2}$, and $z_{3}$ to zero as $t \rightarrow \infty$.

The scalars $c_{1}, c_{2}$, and $c_{3}$ are real positive constants such that the polynomial $p_{2}(s)=$ $s^{3}+c_{3} s^{2}+c_{2} s+c_{1}=0$ is a Hurwitz polynomial.

Let the input-dependent switching surface $\rho(z, u)$ be

$$
\rho(z, u)=u+\frac{1}{g}\left(f+c_{1} z_{1}+c_{2} z_{2}+c_{3} z_{3}\right)
$$

and let $W_{2}$ be a sufficiently large, strictly positive scalar.

The following proposition gives the third result of the paper.

Proposition 5.1. The dynamic control scheme,

$$
u=-\frac{1}{g}\left(f+c_{1} z_{1}+c_{2} z_{2}+c_{3} z_{3}\right)+v
$$

with

$$
\dot{v}=-W_{2} \operatorname{sign}\left(u+\frac{1}{g}\left(f+c_{1} z_{1}+c_{2} z_{2}+c_{3} z_{3}\right)\right),
$$

when applied to the magnetic levitation system (2.5), guarantees the asymptotic convergence of $z_{1}, z_{2}$, and $z_{3}$ to zero as $t \rightarrow \infty$.

Proof. The dynamics in (5.3), (5.4), and (5.5) guarantees the finite-time reachability of $\rho$ to zero from any given initial condition provided that the constant gain $W_{2}$ is chosen to be strictly positive. Moreover, the dynamics in (5.3), (5.4), and (5.5) guarantees that $\rho(z, u) \dot{\rho}(z, u)<0$ (the condition needed to guarantee switching). 
Since $\rho(z, u)$ is driven to zero in finite time, the output $y=z_{1}$ is governed on the sliding surface $(\rho(x, u)=0)$ by the third-order differential equation $y^{(3)}+c_{3} \ddot{y}+c_{2} \dot{y}+c_{1} y=0$. Thus, the output $y(t)=z_{1}$ will converge asymptotically to zero as $t \rightarrow \infty$ because $c_{1}, c_{2}$, and $c_{3}$ are chosen to be positive scalars such that the polynomial $p_{2}(s)=s^{3}+c_{3} s^{2}+c_{2} s+c_{1}$ is a Hurwitz polynomial. Since $z_{1}$ converges to zero, then $z_{2}$ and $z_{3}$ will also converge to zero as $t \rightarrow \infty$.

Using (2.4), it is clear that $x_{1}, x_{2}, x_{3}$ will also converge to their desired values as $t \rightarrow \infty$. Thus, it can be concluded that the dynamic sliding mode controller (5.4), (5.5) guarantees the asymptotic convergence of the states $x_{1}, x_{2}$, and $x_{3}$ to their desired values as $t \rightarrow \infty$.

Remark 5.2. The controller given in Proposition 5.1 can be transformed into the original coordinates of the system by using transformation (2.4). Hence, controller (5.4), (5.5) in the original coordinates is such

$$
u=-\frac{1}{g_{1}}\left(f_{1}+c_{1}\left(x_{1}-x_{1 d}\right)+c_{2} x_{2}+c_{3}\left(g_{c}-\frac{C}{m}\left(\frac{x_{3}}{x_{1}}\right)^{2}\right)\right)+v
$$

with

$$
\dot{v}=-W_{2} \operatorname{sign}\left(u+\frac{1}{g_{1}}\left[f_{1}+c_{1}\left(x_{1}-x_{1 d}\right)+c_{2} x_{2}+c_{3}\left(g_{c}-\frac{C}{m}\left(\frac{x_{3}}{x_{1}}\right)^{2}\right)\right]\right) .
$$

\section{Simulation results of the sliding mode controllers}

Simulations are performed for the static and the two dynamic sliding mode controllers proposed in the paper. The results are shown in this section.

The parameters of the magnetic levitation system are as follows [2]. The coil's resistance $R=28.7 \Omega$, the inductance $L_{1}=0.65 \mathrm{H}$, the gravitational constant $g_{c}=9.81$ milliseconds ${ }^{-2}$, the magnetic force constant $C=1.410^{-4}$, and the mass of the ball $m=11.87 \mathrm{~g}$.

First, the static sliding mode controller (3.3) is applied to the magnetic levitation system (2.3). The parameters of the controller are chosen such that $W=350, \lambda_{1}=61$, and $\lambda_{2}=930$ (which correspond to closed-loop poles of the reduced-order system of -30 and -31). The simulation results are shown in Figure 6.1. The figure shows the position versus time and the control (the applied voltage) versus time for the system when the mass value is nominal and when the mass value is changed by $\pm 25 \%$. It can be seen from the figure that the position converges to its desired value when the mass value is nominal. However, there is a small steady-state error in the position when the mass is changed. Also, some chattering can be seen due to this controller. To further reduce the magnitude of the steady-state error, the value of $W$ can be increased. However, increasing the value of $W$ will lead to a larger control magnitude and more chattering. The value of $W$ was selected so that the magnitudes of the three controllers have similar ranges.

Second, the dynamic sliding mode controller (4.5) is applied to the magnetic levitation system (2.3). The parameters of the controller are chosen such that $\Gamma=50000, m_{1}=93$, $m_{2}=2882$, and $m_{3}=29760$ (which correspond to closed-loop poles of $-30,-31$, and -32). The simulation results are shown in Figure 6.2. The figure shows the position versus time and the control versus time for the system when the mass value is nominal and 
102 Sliding mode control of a magnetic levitation system

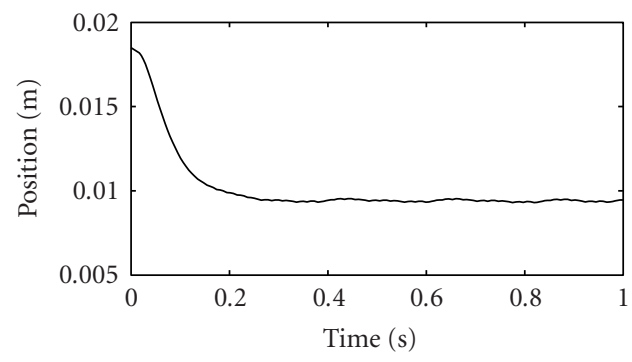

(a)

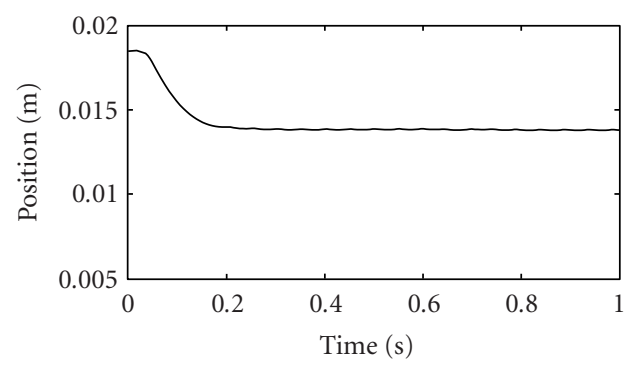

(c)

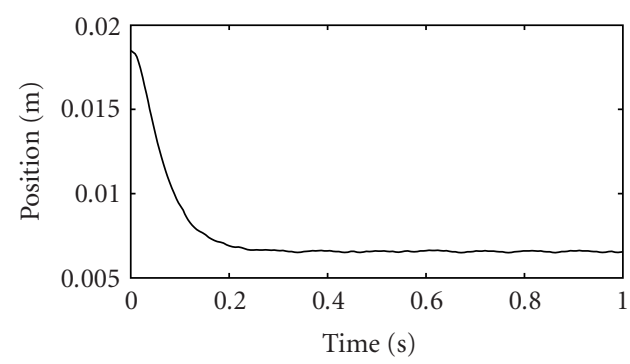

(e)

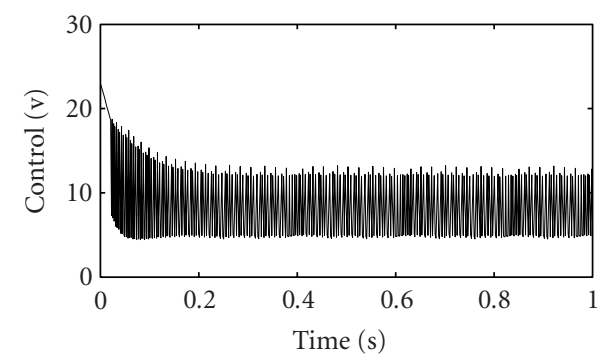

(b)

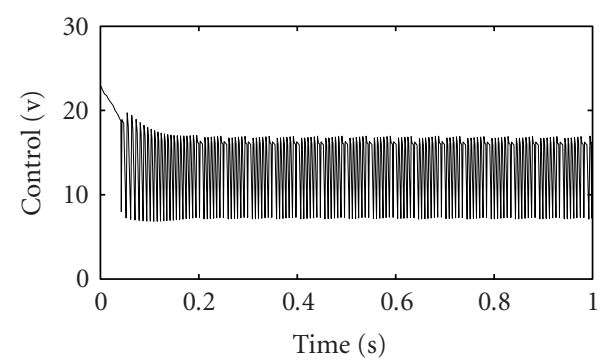

(d)

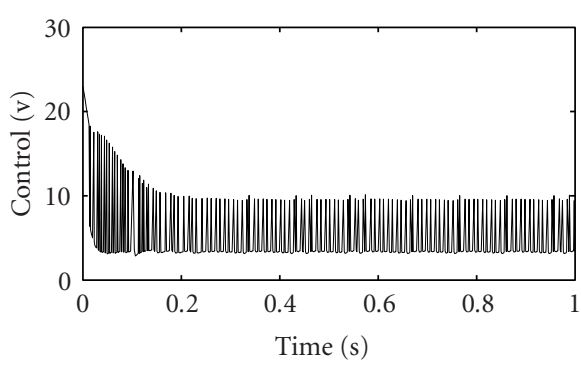

(f)

Figure 6.1. The position and the control versus time when using the static sliding mode scheme. (a) Position for $m=11.87 \mathrm{~g}$, (b) control for $m=11.87 \mathrm{~g}$, (c) position for $m=11.87 \mathrm{~g}+25 \%$, (d) control for $m=11.87 g+25 \%$, (e) position for $m=11.87 g-25 \%$, and (f) control for $m=$ $11.87 g-25 \%$.

when the mass value is changed by $\pm 25 \%$. It can be seen from Figure 6.2 that the position converges to its desired value even when the mass of the object varies by $\pm 25 \%$. Hence, the controlled system is robust to changes in the mass value. Also, it can be seen from the figure that the chattering in the control signal is greatly reduced when the dynamic sliding mode controller is applied.

Third, the modified dynamic sliding mode controller (5.6), (5.7) is applied to the magnetic levitation system (2.3). The parameters of the controller are chosen such that $W_{2}=100, c_{3}=93, c_{2}=2882$, and $c_{1}=29760$ (which correspond to closed-loop poles 


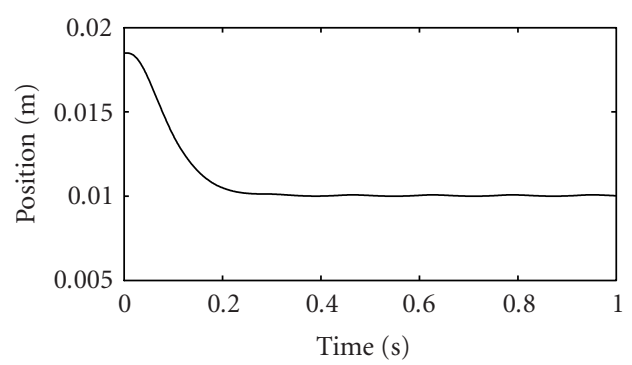

(a)

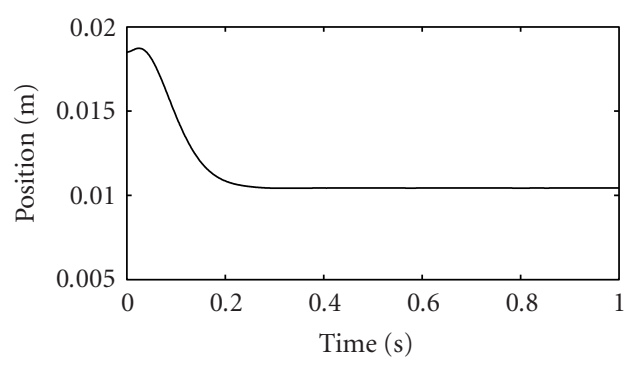

(c)

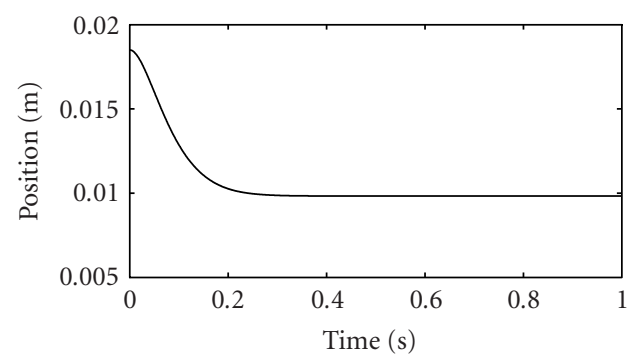

(e)

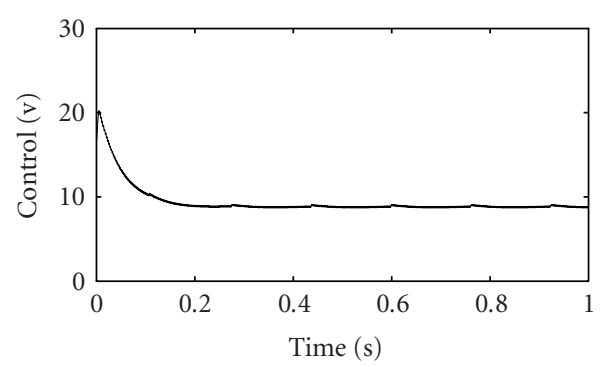

(b)

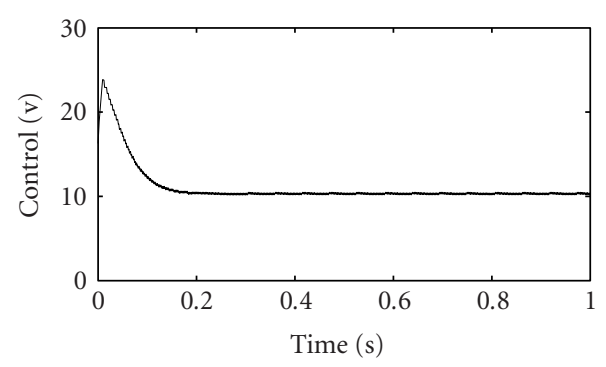

(d)

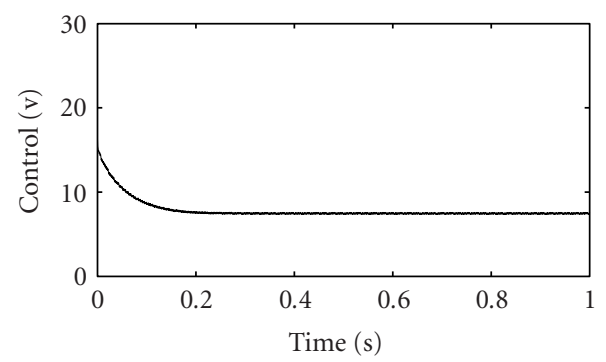

(f)

Figure 6.2. The position and the control versus time when using the dynamic sliding mode scheme. (a) Position for $m=11.87 \mathrm{~g}$, (b) control for $m=11.87 \mathrm{~g}$, (c) position for $m=11.87 \mathrm{~g}+25 \%$, (d) control for $m=11.87 g+25 \%$, (e) position for $m=11.87 g-25 \%$, and (f) control for $m=$ $11.87 g-25 \%$.

of $-30,-31$, and -32 ). The simulation results are shown in Figure 6.3. The figure shows the position versus time and the control versus time for the system when the mass value is nominal and when the mass value is changed by $\pm 25 \%$. It can be seen from Figure 6.3 that the position converges to its desired value even when the mass of the object varies by $\pm 25 \%$. Hence, the controlled system is robust to changes in the mass value. Also, it can be seen from the figure that the chattering in the control signal is almost eliminated when applying the modified dynamic sliding mode controller. Finally, Figure 6.4 shows 
104 Sliding mode control of a magnetic levitation system

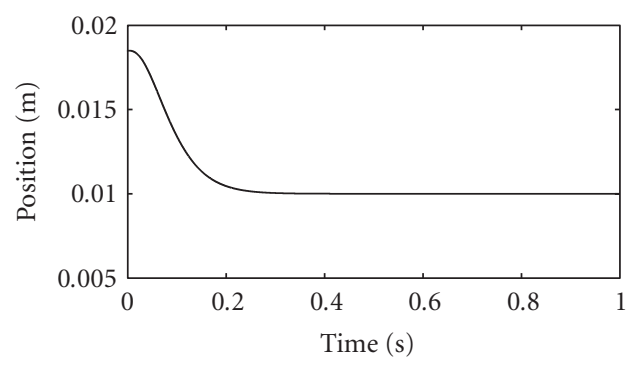

(a)

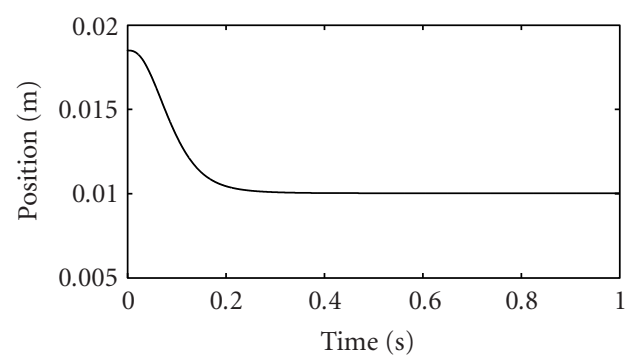

(c)

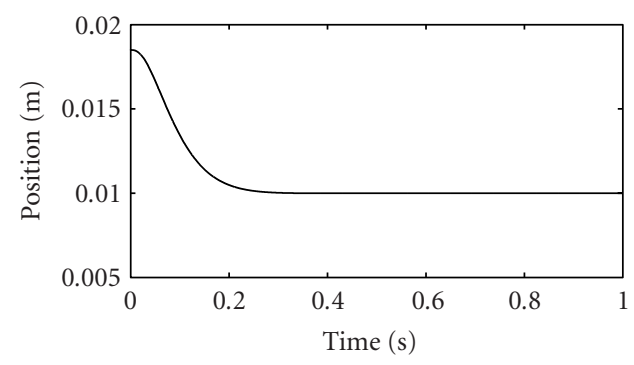

(e)

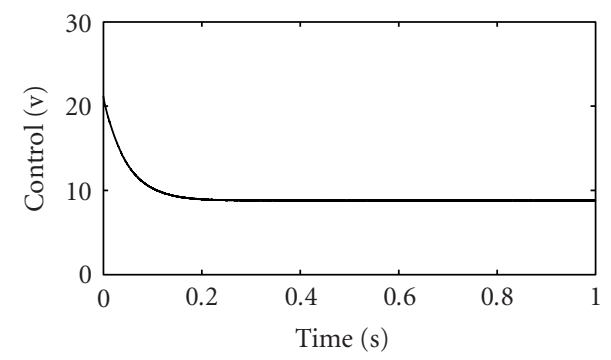

(b)

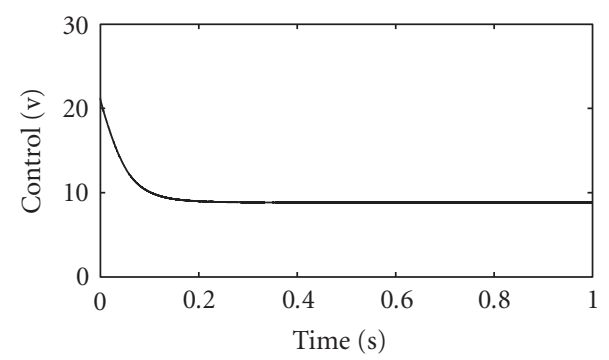

(d)

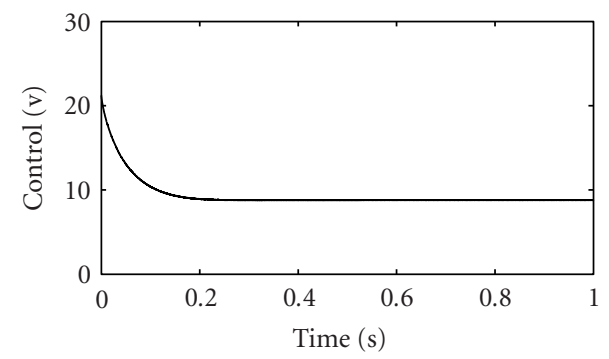

(f)

Figure 6.3. The position and the control versus time when using the modified dynamic sliding mode scheme. (a) Position for $m=11.87 \mathrm{~g}$, (b) control for $m=11.87 \mathrm{~g}$, (c) position for $m=11.87 \mathrm{~g}+$ $25 \%$, (d) control for $m=11.87 g+25 \%$, (e) position for $m=11.87 g-25 \%$, and (f) control for $m=11.87 g-25 \%$.

the position versus time for the three proposed controllers for the case when the mass value is nominal.

Therefore, the simulation results indicate that the proposed control schemes work well when applied to the magnetic levitation system. It can be concluded from the simulations that the static control scheme is somewhat robust to changes in the mass of the object. However, the dynamic controllers are very robust. It is also clear that the dynamic controllers greatly reduce the chattering. 


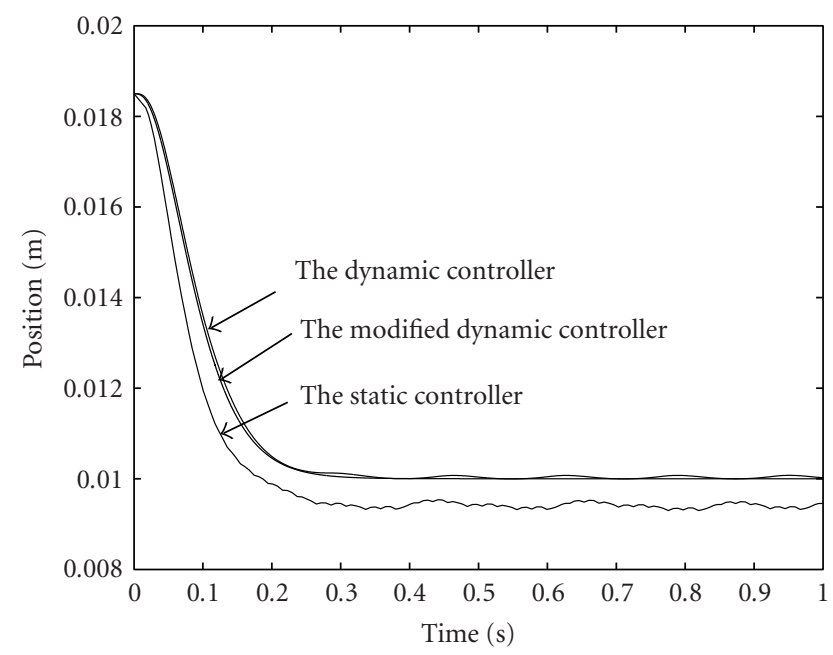

Figure 6.4. The position versus time for the three proposed controllers.

\section{Conclusion}

The problem of static and dynamic SMC of a magnetic levitation system is addressed in this paper. A static SMC scheme is derived first. To reduce the chattering problem, two dynamic sliding mode controllers are designed. Simulation results of the proposed control schemes are given to show the effectiveness of these controllers. Moreover, the robustness of the developed control schemes to variations in the parameters of the system is investigated. It is found that the three control schemes are robust to parameter variations. However, the third control scheme (the modified dynamic sliding mode scheme) gives the best results among the three controllers. Future work will address the experimental implementation of the proposed control schemes.

\section{References}

[1] P. Allaire and A. Sinha, Robust sliding mode control of a planar rigid rotor system on magnetic bearings, Proc. 6th International Symposium on Magnetic Bearings, (Massachusetts), August 1998, pp. 577-586.

[2] W. Barie and J. Chiasson, Linear and nonlinear state-space controllers for magnetic levitation, International Journal of Systems Science 27 (1996), no. 11, 1153-1163.

[3] G. Bartolini and T. Zolezzi, Variable structure systems nonlinear in the control law, IEEE Trans. Automatic Control 30 (1985), no. 7, 681-684.

[4] N. N. Bengiamin and W. C. Chan, Variable structure control of electric power generation, IEEE Transactions on Power Apparatus and Systems 101 (1982), no. 2, 376-380.

[5] G. D. Buckner, Intelligent bounds on modeling uncertainties: applications to sliding mode control of a magnetic levitation system, IEEE International Conference on Systems, Man, and Cybernetics, (Arizona), vol. 1, 2001, pp. 81-86.

[6] A. Charara, J. De Miras, and B. Caron, Nonlinear control of a magnetic levitation system without premagnetization, IEEE Transactions on Control Systems Technology 4 (1996), no. 5, 513523. 
[7] M.-Y. Chen, C.-C. Wang, and L.-C. Fu, Adaptive sliding mode controller design of a dual-axis maglev positioning system, Proc. 2001 American Control Conference, (Virginia), vol. 5, 2001, pp. 3731-3736.

[8] D. Cho, Y. Kato, and D. Spilman, Sliding mode and classical controllers in magnetic levitation systems, IEEE Control Systems Magazine 13 (1993), no. 1, 42-48.

[9] A. El Hajjaji and M. Ouladsine, Modeling and nonlinear control of magnetic levitation systems, IEEE Transactions on Industrial Electronics 48 (2001), no. 4, 831-838.

[10] O. El Rifai and K. Youcef-Toumi, Achievable performance and design trade-offs in magnetic levitation control, Proc. 5th International Workshop on Advanced Motion Control (AMC '98), (Coimbra, Portugal), July 1998, pp. 586-591.

[11] M. R. Filho and C. J. Munaro, A design methodology of tracking controllers for magnetic levitation systems, Proc. 2001 IEEE International Conference on Control Applications, (Mexico City), 2001, pp. 47-51.

[12] M. Fujita, F. Matsumura, and K. Uchida, Experiments on the $H^{\infty}$ disturbance attenuation control of a magnetic suspension system, Proc. 29th IEEE Conference on Decision and Control, (Hawaii), vol. 5, December 1990, pp. 2773-2778.

[13] M. Fujita, T. Namerikawa, F. Matsumura, and K. Uchida, $\mu$-synthesis of an electromagnetic suspension system, IEEE Trans. Automatic Control 40 (1995), no. 3, 530-536.

[14] S. A. Green and K. C. Craig, Robust, design, nonlinear control of magnetic-levitation systems, Journal of Dynamics, Measurement, and Control 120 (1998), no. 4, 488-495.

[15] H. Hashimoto, H. Yamamoto, S. Yanagisawa, and F. Harashima, Brushless servo motor control using variable structure approach, IEEE Transactions on Industry Applications 24 (1988), no. $1,160-170$.

[16] D. M. M. Hassan and A. M. Mohamed, Variable structure control of a magnetic levitation system, Proc. 2001 American Control Conference, (Virginia), vol. 5, 2001, pp. 3725-3730.

[17] J. K. Hedrick and S. Gopalswamy, Nonlinear flight control design via sliding methods, Journal of Guidance, Control and Dynamics 13 (1990), no. 5, 850-858.

[18] C.-M. Huang, J.-Y. Yen, and M.-S. Chen, Adaptive nonlinear control of repulsive maglev suspension systems, Control Engineering Practice 8 (2000), no. 5, 1357-1367.

[19] J. Y. Hung, W. B. Gao, and J. C. Hung, Variable structure control: a survey, IEEE Transactions on Industrial Electronics 40 (1993), no. 1, 2-22.

[20] J. C. Kantor, Nonlinear sliding-mode controller and objective function for surge tanks, Internat. J. Control 50 (1989), no. 5, 2025-2047.

[21] Y. C. Kim and H. K. Kim, Gain scheduled control of magnetic suspension systems, Proc. American Control Conference, (Maryland), vol. 3, July 1994, pp. 3127-3131.

[22] M. Lairi and G. Bloch, A neural network with minimal structure for maglev system modeling and control, Proc. 1999 IEEE International Symposium on Intelligent Control/Intelligent Systems and Semiotics, (Massachusetts), 1999, pp. 40-45.

[23] A. M. Mohamed, F. Matsumura, T. Namerikawa, and J.-H. Lee, Q-parametrization/ $\mu$-control of an electromagnetic suspension system, Proc. 1997 IEEE International Conference on Control Applications, (Connecticut), October 1997, pp. 604-608.

[24] K. Nonami and K. Nishina, Robust control of magnetic bearing systems by means of sliding mode control, Proc. 3rd International Symposium on Magnetic Bearings, (Virginia), 1992, pp. 221-226.

[25] A. Rundell, S. Drakunov, and R. DeCarlo, A sliding mode observer and controller for stabilization of rotational motion of a vertical shaft magnetic bearing, IEEE Transactions on Control Systems Technology 4 (1996), no. 5, 598-608.

[26] H. Sira-Ramírez, On the sliding mode control of multivariable nonlinear systems, Internat. J. Control 64 (1996), no. 4, 745-765. 
[27] H. Sira-Ramírez, S. Ahmad, and M. Zribi, Dynamical feedback control of robotic manipulators with joint flexibility, IEEE Transactions on Systems, Man and Cybernetics 22 (1992), no. 4, 736-747.

[28] H. Sira-Ramírez, O. Llanes-Santiago, and N. Arrieta Fernández, On the stabilization of nonlinear systems via input-dependent sliding surfaces, Internat. J. Robust Nonlinear Control 6 (1996), no. 8, 771-780.

[29] J. J. E. Slotine and W. Li, Applied Nonlinear Control, Prentice-Hall, New Jersey, 1991.

[30] D. L. Trumper, M. Olson, and P. K. Subrahmanyan, Linearizing control of magnetic suspension systems, IEEE Transactions on Control Systems Technology 5 (1997), no. 4, 427-438.

[31] V. I. Utkin, Variable structure systems with sliding modes, IEEE Trans. Automatic Control 22 (1977), no. 2, 212-222.

[32] Sliding Modes and Their Application in Variable Structure Systems, Mir Publishers, Moscow, 1978.

[33] Sliding Modes in Control and Optimization, Communications and Control Engineering Series, Springer-Verlag, Berlin, 1992.

[34] - Variable structure systems and sliding mode — state of the art assessment, Variable Structure Control for Robotics and Aerospace Applications (K. D. Young, ed.), Elsevier, 1993, pp. 9-32.

[35] Z.-J. Yang and M. Tateishi, Robust nonlinear control of a magnetic levitation system via backstepping approach, Proc. 37th SICE Annual Conference (SICE '98), (Chiba, Japan), July 1998, pp. 1063-1066.

[36] K. D. Young (ed.), Variable Structure Control for Robotics and Aerospace Applications, Elsevier Science, New York, 1993.

[37] K. D. Young, V. I. Utkin, and U. Ozguner, A control engineer's guide to sliding mode control, IEEE Transactions on Control Systems Technology 7 (1999), no. 3, 328-342.

[38] F. Zhang and K. Suyama, Nonlinear feedback control of magnetic levitating system by exact linearization approach, Proc. 4th IEEE Conference on Control Applications, (New York), September 1995, pp. 267-268.

[39] F. Zhao, S. C. Loh, and J. A. May, Phase-space nonlinear control toolbox: the maglev experience, 5th International Hybrid Systems Workshop, (Ind, September 1997) (P. Antsaklis, W. Kohn, M. Lemmon, A. Nerode, and S. Sastry, eds.), Lecture Notes in Computer Science, vol. 1567, Springer-Verlag, London, 1999, pp. 429-444.

[40] F. Zhao and R. Thornton, Automatic design of a Maglev controller in state space, Proc. of the 31st Conference on Decision and Control, (Tucson, Ariz), December 1992, pp. 2562-2567.

[41] A. S. I. Zinober, Deterministic Control of Uncertain Systems, IEE Control Engineering Series, vol. 40, Peter Peregrinus, London, 1990.

[42] - Variable Structure and Lyapunov Control, Lecture Notes in Control and Information Sciences, vol. 193, Springer-Verlag, London, 1994.

N. F. Al-Muthairi: Department of Electrical Engineering, Kuwait University, P.O. Box 5969, Safat13060, Kuwait

E-mail address: muthairi@eng.kuniv.edu.kw

M. Zribi: Department of Electrical Engineering, Kuwait University, P.O. Box 5969, Safat-13060, Kuwait

E-mail address: mzribi@eng.kuniv.edu.kw 


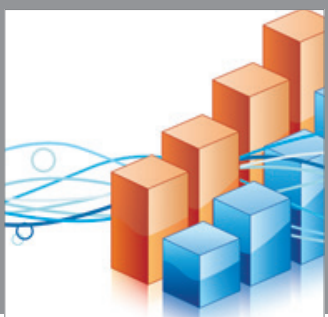

Advances in

Operations Research

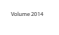

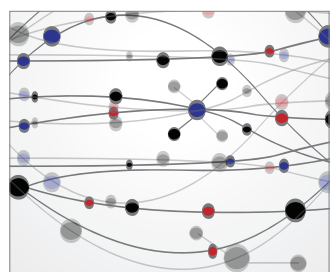

\section{The Scientific} World Journal
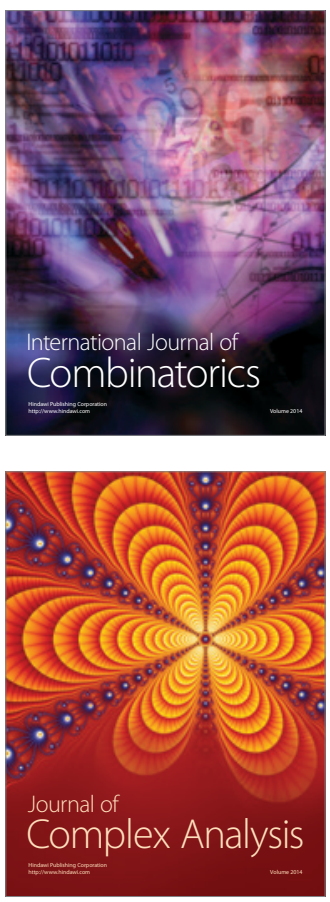

International Journal of

Mathematics and

Mathematical

Sciences
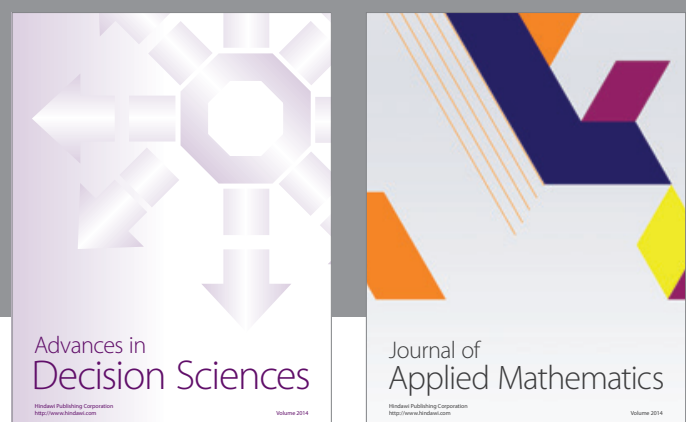

Journal of

Applied Mathematics
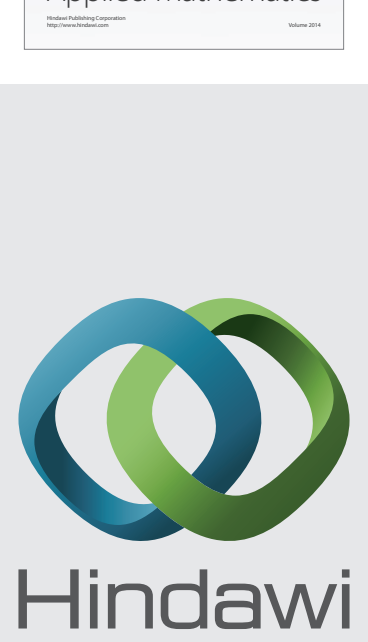

Submit your manuscripts at http://www.hindawi.com
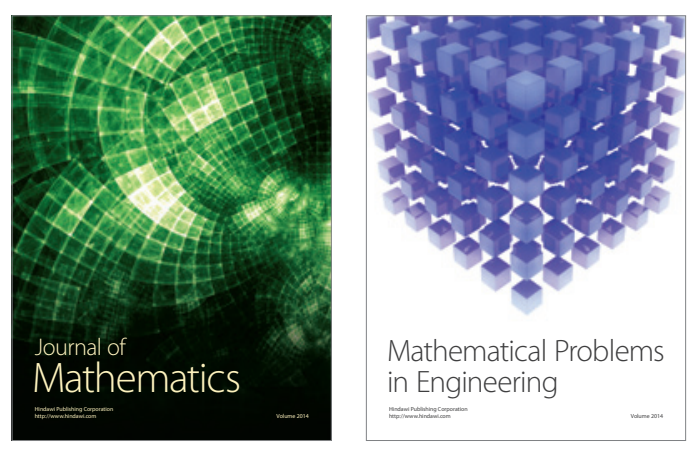

Mathematical Problems in Engineering
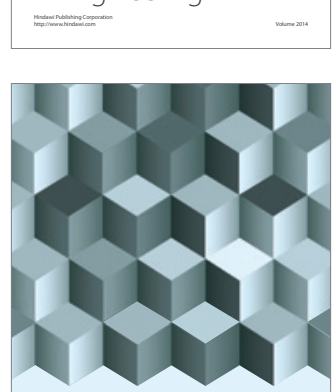

Journal of

Function Spaces
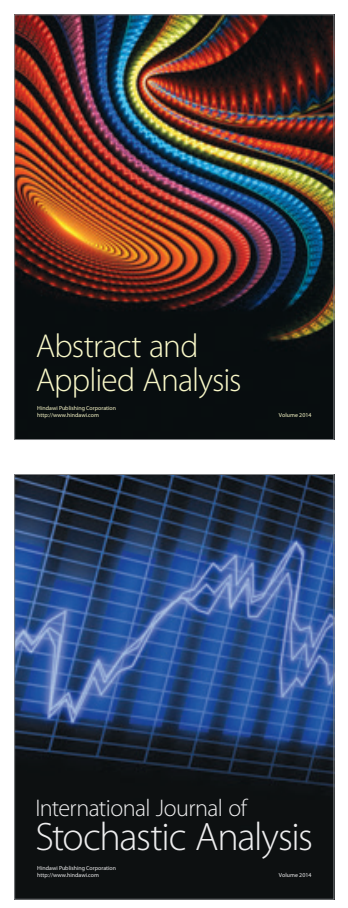

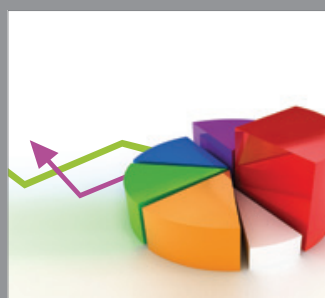

ournal of

Probability and Statistics

Promensencen
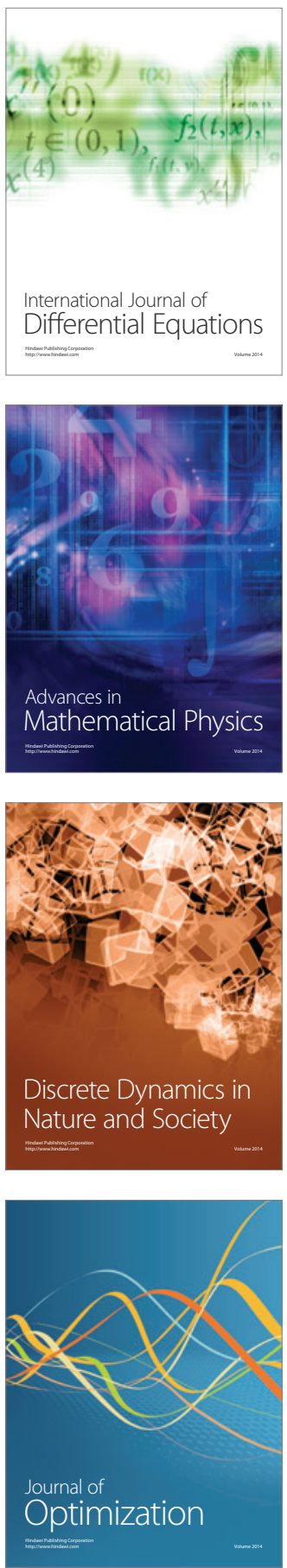